\title{
Cross-Sectional Study of HPV Self-Sampling among Indian Women-A Way Forward
}

\author{
Kavita V. Anand ${ }^{1}$ Gauravi A. Mishra ${ }^{10}$ Sharmila A. Pimple ${ }^{10}$ Sanjay K. Biswas ${ }^{2}$ \\ Vasundhara Y. Kulkarni ${ }^{1}$ Gaurav Salunke ${ }^{2(1)}$ \\ ${ }^{1}$ Department of Preventive Oncology, Centre for Cancer \\ Epidemiology, Tata Memorial Centre, Homi Bhabha National \\ Institute, Mumbai, Maharashtra, India \\ 2 Department of Microbiology, Tata Memorial Centre, Homi Bhabha \\ National Institute, Mumbai, Maharashtra, India

\begin{abstract}
Address for correspondence Gauravi Mishra, MD, PSM, Department of Preventive Oncology, Centre for Cancer Epidemiology, Tata Memorial Centre, Homi Bhabha National Institute, Room No. 314, 3rd Floor, Service Block, E. Borges Marg, Parel, Mumbai 400 012, Maharashtra, India
\end{abstract} \\ (e-mail: gauravi2005@yahoo.co.in; mishraga@tmc.gov.in)
}

Ind J Med Paediatr Oncol 2022;43:103-108.

\begin{abstract}
Keywords

- cervical cancer screening

- HPV test

- HPV self-sampling

- art-based strategy

- low-resource settings

Molecular human papillomavirus (HPV) DNA is a recommended test for any country planning cervical cancer screening as a national policy. The emerging literature proposes HPV self-sampling (HPV-SS) as a feasible implementing strategy in lowincome settings. The success of this strategy would depend on developing impactful health education materials, understanding modalities toward generating awareness, and precision in performing the screening test among beneficiaries. The current paper is an interim analysis of ongoing research undertaken to understand the acceptability of HPV-SS among Indian women across different community settings. The study design has two modalities for generating awareness: (1) health education arm wherein the awareness and steps of collecting self-sample are explained by health personnel, and (2) the pamphlet arm wherein pictorial illustrations depicting the steps to conduct HPV-SS are distributed among women. The quality of samples is compared with primary health worker samples (PHW-S). Initial results of this study support the acceptance of HPV-SS (97\%) among urban slum settings. An agreement between HPV-SS and PHW-S was demonstrated to be $95.1 \%$. The results of the pamphlet arm were comparable to the health education arm in every aspect. The art-based strategy seems like a promising communication modality for generating awareness toward cervical cancer screening using HPV-SS in low-resource settings.
\end{abstract}

\section{Introduction}

India reports a high burden of cervical cancer. ${ }^{1,2}$ The factors contributing to a high burden of disease are the lack of health care infrastructure and access to available facilities. Among social determinants, low literacy level, lack of awareness of cervical cancer symptoms and lack of priority of women's health issues are some of the contributing factors. ${ }^{3}$ These factors together have a potential to lead to a global disparity in terms of population coverage for cervical cancer screening in India. A population-based survey conducted across 57 countries reported cervical cancer screening coverage of $19 \%$ on an average in developing countries compared with
DOI https://doi.org/ 10.1055/s-0042-1742642. ISSN 0971-5851. (c) 2022. Indian Society of Medical and Paediatric Oncology. All rights reserved.

This is an open access article published by Thieme under the terms of the Creative Commons Attribution-NonDerivative-NonCommercial-License, permitting copying and reproduction so long as the original work is given appropriate credit. Contents may not be used for commercial purposes, or adapted, remixed, transformed or built upon. (https://creativecommons.org/ licenses/by-nc-nd/4.0/)

Thieme Medical and Scientific Publishers Pvt. Ltd., A-12, 2nd Floor, Sector 2, Noida-201301 UP, India 
$63 \%$ in developed countries. The author commented that older and poor women, who are at the highest risk of developing cervical cancer, are least likely to be screened. ${ }^{4}$

Currently, India does not have an organized screening program. Although visual inspection with acetic acid (VIA) is a recommended screening test, it is challenging to sustain and scale up the program due to repeated training, quality control, and screening rounds needed. ${ }^{5}$ To avail VIA test, women need to visit health care facilities with trained health personnel to perform the test. This scenario represents programmatic barriers for screening in terms of accessibility and human resources. Currently, there is sufficient evidence from epidemiological studies about the causal role of persistent oncogenic human papillomavirus (HPV) infection and cervical cancer. ${ }^{6,7}$ The direct detection of HPV in cervical specimens by a molecular test can offer an alternative for cytology/VIA-based screening. Among the various emerging HPV molecular tests, the hybrid capture-2 test (HC2) has been extensively evaluated in various health care settings. ${ }^{8,9}$ The test identifies women at the risk of developing cervical cancer, needing a close follow-up. ${ }^{10,11}$ The advantages of HPV-DNA molecular test are high sensitivity (reported from developing countries), longer screening intervals if tested negative, the potential of self-collection, reproducibility, and standardization of the test. ${ }^{10,12,13}$ HPV self-sampling (HPV$\mathrm{SS}$ ) is posed to be a viable option to increase the uptake of cervical cancer screening among women. At the national level implementation strategy, the test has the additional advantage of reducing the human resources in terms of empowering the women to undertake their own test. ${ }^{14}$

Among all the above factors, the current study mainly explores the potential of acceptance of HPV-SS as a preferred screening method by Indian women. India is a country with diversities. Promoting HPV-SS shall need effective information, education, and communication materials (IEC) in generating awareness toward screening and performing the HPV-SS test with confidence among the beneficiaries. This is crucial in settings where illiteracy is a key barrier in undertaking cervical cancer screening tests.

The current paper discusses interim results of the main study in terms of acceptability of self-sampling as a screening modality to increase the coverage for cervical cancer screening, the impact of communication modalities of IEC materials (the health education arm versus pamphlet arm) to increase the uptake of HPV-SS, and quality of HPV-SS measured in terms of agreement rates between the HPV-SS and primary health care worker collected HPV samples (HPV-PHW) among women in the slum settings in Mumbai, Maharashtra.

Quality of HPV-SS measured in terms of agreement rates between the self-sampling and HPV-PHW among women in the slum setting was considered as a primary objective of the study.

\section{Materials and Methods}

\section{Inclusion Criteria}

Inclusion criteria were sexually active non-pregnant women in the age group of 30 to 55 years with no previous history of cervical cancer.

\section{Exclusion Criteria}

Exclusion criteria were women not sexually active, who underwent total hysterectomy, with prior treatment history for cervical cancer, and pregnant or postpartum 6 months.

\section{Study Design}

The main study was a cross-sectional study proposed to be implemented across three communities: namely (1) urban slum area, (2) urban non-slum area, and (3) rural area (-Fig. 1). Herein, we tried to understand if HPV-SS would be similarly or preferentially accepted by specific groups. For each setting, three clusters were identified and one of the clusters under each setting was selected by a lottery method for implementation. The current study presents the results of urban slum community settings collected during the study period of 12th April 2021 to 24th June 2021.

As per our hypothesis, the modality of communication may influence cervical cancer screening uptake and quality of self-sampling. To address this, the current study was designed with two interventional arms-(1) health education arm and (2) pamphlet arm. Each selected cluster was then demarcated for two interventions using geographical landmarks. In the health education arm, primary awareness for cervical cancer (risk factors, types of screening tests, and advantage of early diagnosis and prevention) and steps demonstrating the method to collect HPV-SS was explained in the local language using flip charts/posters by medical social workers (MSW). In the pamphlet arm, art-based strategy pamphlets with self-explanatory pictorial illustrations demonstrating the steps of the procedure of collecting HPV-SS were distributed by MSW.

\section{Methodology}

As per the literature, the prevalence of HPV in self-sample is assumed to be 7 to $10 \%$. To get a concordance rate of $80 \%$, at $95 \%$ confidence interval $(\mathrm{CI})$ and power of $80 \%$, estimated sample size comprised 1,600 women (500 under urban slum settings, 500 under urban non-slum settings, and 600 under rural settings) to undergo HPV testing by both the methods for the main study.

Under the current study, a total of 500 women, 250 women in the health education arm and 250 women in the pamphlet arm, were enrolled. After the detailed mapping of the area, door-to-door survey was conducted by trained MSWs to register eligible women and invite them to participate in the study. Eligible women were enrolled following informed consent, and the study questionnaires were completed.

After the awareness session as per the arm assigned, HPVSS kits were distributed to women in both groups. Used/unused kits were collected either on the same day or within 72 hours by the MSWs. All women enrolled in the study were invited for HPV-PHW samples. The women who tested positive on either HPV tests were referred to the tertiary care center for further diagnostic workup.

The MSWs and the PHWs recruited under this trial received intensive training on delivering the standard 


\section{Study Algorithm (Figure 1)}

Selecting three sub-groups of population: Urban shum (500) \& non slum areas (500) in Mumbai \& Rural area of Raigad/Thane district (600)

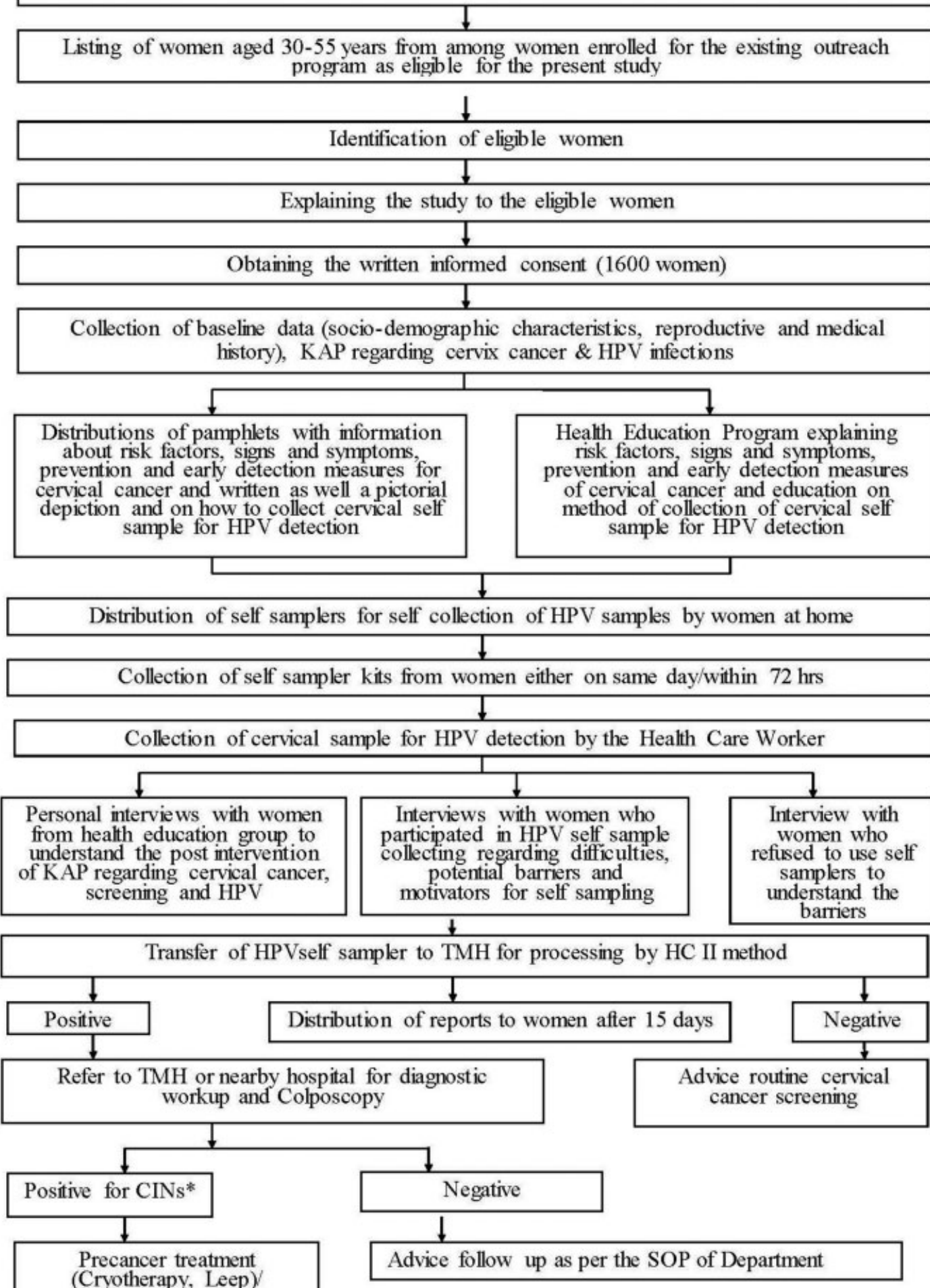

(Cryotherapy, Leep)

Treatment for cancer

Fig. 1 Demonstrates the detailed methodology of study. ${ }^{*}$ Cervical intraepithelial neoplasia. 
health education on cervical cancer for 4 weeks. MSWs were additionally trained for conducting survey, mapping, and HPV-SS test. PHWs received hands-on training in collecting HPV samples using the HC2 test for an additional period of 4 weeks in the institutional department as per the standard operating protocols (SOPs). The audit for quality control was planned every month.

\section{Screen Positivity Criteria for the HC2 Test}

To diagnose HPV infection, samples were processed by the HC2 test which has probes for 13 recognized oncogenic HPV types. The relative light unit/cut-off ratio $\geq 1$, corresponding to 5,000 or more copies of virus in a sample, is considered a positive test. The transportation of the samples from the field to institute was as per the SOPs of the Institutional Department of Microbiology. 8,9

\section{Statistical Analysis}

The quantitative data related to the study's objective were captured and analyzed using IBM SPSS statistics for windows, version 25.0 (IBM Corp, Armok, NY, United States). For the current paper, agreement between the HPV-SS and HPVPHW samples using the molecular HPV test was analyzed using Kappa statistics for the probability of chance along with $95 \% \mathrm{CI}$.

\section{Ethics}

The female participants were enrolled only after informed written consent was obtained. The study was conducted in accordance with the Declaration of Helsinki and Good Clinical Practices as defined by the International Conference on Harmonization. The study received clearance of the Tata Memorial Hospital Institutional Review Board on 2nd March 2017, Project No- 1686.

\section{Results}

The overall acceptance of HPV-SS was 97\% (485) in the target population (-Table 1). The acceptance of HPV-SS was $100 \%$ (250) in the health education arm and 94\% (235) in the pamphlet arm for all those receiving the instructions in both formats. The agreement between the HPV-PHW sample and the HPV-SS was demonstrated to be $94.69 \%$ (232) and 95.61\% (196) in the health education arm and pamphlet arm, respectively. Overall, the agreement rate was $95.11 \%$ (428). The kappa value demonstrated moderate agreement between the two samples readings by the HC2 test, $k=0.55$ (95\% $\mathrm{CI}=0.34-0.77), p<0.001$.

\section{Discussion}

In India, cervical cancer is a vivid indicator of health inequality within the country with the burden of disease reported high among rural and low-income areas. ${ }^{15}$ Hence, all efforts should be made to cover this hard-toreach population to reduce the incidence and mortality rates for the country. The current article reports interim data on acceptability and quality of HPV-SS among women in urban slum settings using a camp-based approach. Only slum women were selected for the current paper as they face challenges such as non-availability of washrooms in the household and lack of privacy for collecting selfsamples.

This setting was high risk for HPV infections in terms of low economy, risky behavior, sharing common toilets, and low vaginal hygiene. The women enrolled in the study were in the median age group of 38 years which is a recognized age for the persistence of oncogenic HPV infection. A single round of screening in this age group has the advantage of prevention and downstaging of the disease. ${ }^{14,15}$ The study overall demonstrated high acceptability (97\%) of HPV-SS among this group of women (-Table $\mathbf{1}$ ).

Table 1 Acceptability and agreements between HPV self-sampling and health worker collected HPV samples between the health education arm and pamphlet education arm as modalities of communications

\begin{tabular}{|c|c|c|c|c|c|c|c|c|c|c|}
\hline \multicolumn{2}{|l|}{ Variable } & \multicolumn{3}{|c|}{$\begin{array}{l}\text { Health education arm } \\
n=250\end{array}$} & \multicolumn{3}{|c|}{$\begin{array}{l}\text { Pamphlet education } \\
\text { arm } n=250\end{array}$} & \multicolumn{3}{|c|}{ Total $n=500$} \\
\hline \multicolumn{11}{|l|}{ 1. a. Acceptance of self-sampling } \\
\hline \multicolumn{2}{|l|}{ Acceptance of self-sampling $n(\%)$} & \multicolumn{3}{|c|}{$250(100 \%)$} & \multicolumn{3}{|c|}{$235(94 \%)$} & \multicolumn{3}{|c|}{$485(97 \%)$} \\
\hline \multicolumn{11}{|l|}{ 1. b. Agreements between samples } \\
\hline & & \multicolumn{3}{|c|}{$\begin{array}{l}\text { Health worker collected } \\
\text { sample }\end{array}$} & \multicolumn{3}{|c|}{$\begin{array}{l}\text { Health worker } \\
\text { collected sample }\end{array}$} & \multicolumn{3}{|c|}{$\begin{array}{l}\text { Health worker collected } \\
\text { sample }\end{array}$} \\
\hline \multicolumn{2}{|l|}{ HPV test result } & + ve & - ve & Total & + ve & - ve & Total & + ve & - ve & Total \\
\hline \multirow[t]{3}{*}{ Women self- collected sample } & + ve & 9 & 7 & 16 & 7 & 4 & 11 & 16 & 11 & 27 \\
\hline & - ve & 6 & 223 & 229 & 5 & 189 & 194 & 11 & 412 & 423 \\
\hline & Total & 15 & 230 & $245^{a}$ & 12 & 193 & $205^{a}$ & 27 & 423 & $450^{a}$ \\
\hline \multicolumn{2}{|l|}{ Agreement } & \multicolumn{3}{|c|}{$232(94.69 \%)$} & \multicolumn{3}{|c|}{$196(95.61 \%)$} & \multicolumn{3}{|c|}{$428(95.11 \%)$} \\
\hline \multicolumn{2}{|l|}{ Kappa $(95 \% \mathrm{Cl})$} & \multicolumn{3}{|c|}{$0.55(0.34-0.77)$} & \multicolumn{3}{|c|}{$0.58(0.34-0.83)$} & \multicolumn{3}{|c|}{$0.57(0.40-0.73)$} \\
\hline
\end{tabular}

Abbreviations: HPV, human papilloma virus; -ve, negative for HPV in cervical sample; + ve, positive for HPV in cervical sample.

${ }^{a}$ Women gave self-collected sample and health worker sample was also collected. 
Moreover, beyond acceptance of the test, it is crucial that women perform the test with precision. There are numerous studies that have reported comparable performance of self-sampling to clinician collected samples. ${ }^{16,17}$ To our knowledge, this is the first study that compares the performance between the self-collected and trained PHWs collected HPV samples. Our study also demonstrates comparable agreements between HPV-SS and HPV-PHW samples. Understanding that the majority of Indian population resides in rural areas with illiteracy and social stigmatization ${ }^{18}$ being a major barrier in taking the test, it becomes important to understand, develop, and evaluate appropriate IEC materials to raise awareness toward cervical cancer and the screening test to be implemented. The educative materials also need to be effective in de-stigmatizing and facilitating the undertaking of screening. Prior knowledge of the screening test helps women to do so confidently. Currently, resources are needed for one-to-one counseling for women before preparing them to undertake HPV-SS. ${ }^{16,17,19}$ This strategy may not be feasible in humanresource-constrained countries like India. The art-based health promotion strategies have been proven effective in facilitating and mobilizing women among diverse settings. These strategies are warranted in low-resource settings. ${ }^{19,20}$ The strength of our study design is the use of two modalities of communication in two groups, which shall help us understand the effective communication modality. The current study demonstrates an equally good acceptance of HPV-SS by women in the pamphlet arm comparable to the health education arm ( - Table $\mathbf{1}$ ). Recruiting and training MSWs and PHWs as project staff for implementation shall help us understand the time needed for capacity building and quality of deliverables with the goal of transferrable skills. This is an attempt to understand the feasibility of integrating HPV-SS in public health systems.

\section{Conclusion}

The initial findings of the study support the acceptability of HPV-SS as a primary screening test in the targeted population. In resources constraint settings, the current finding demonstrates the potential of art-based strategy as a modality in facilitating HPV-SS among women. The study also supports the feasibility of rapidly training para-medical staff in implementing the test at ground levels. These findings are encouraging toward building a sustainable and operational cervical cancer screening model for the country.

\section{Work to be Credited}

This work is credited to the Department of Preventive Oncology, Centre for Cancer Epidemiology (CCE), Tata Memorial Centre, Homi Bhabha National Institute (HBNI), Mumbai.

\section{Approval by all Authors}

This manuscript has been read and approved by all the authors and represents honest work.
CTRI No

CTRI/2020/09/027748.

Funding

This work was supported by the Terry Fox Foundation, Burnaby, British Columbia V5A4Y6.

\section{Conflict of Interest}

None declared.

\section{Acknowledgment}

The authors would like to thank Mr. Anil S. Patil for helping with statistical data analysis, Dr. Kshama R.Vairagi and Mr. Tushar M. Jadhav for their support in developing art-based education materials under the study. We thank Ms. Preethi Vijayan and Mr. Ravi K. Ubhare for their practical work.

\section{References}

1 Ferlay J, Colombet M, Soerjomataram I, et al. Estimating the global cancer incidence and mortality in 2018: GLOBOCAN sources and methods. Int J Cancer 2019;144(08):1941-1953

2 Bray F, Ferlay J, Soerjomataram I, Siegel RL, Torre LA, Jemal A. Global cancer statistics 2018: GLOBOCAN estimates of incidence and mortality worldwide for 36 cancers in 185 countries. CA Cancer J Clin 2018;68(06):394-424

3 Mishra GA, Pimple SA, Shastri SS. Prevention of cervix cancer in India. Oncology 2016;91(Suppl 1):1-7

4 Gakidou E, Nordhagen S, Obermeyer Z. Coverage of cervical cancer screening in 57 countries: low average levels and large inequalities. PLoS Med 2008;5(06):e132

5 Arbyn M, Sankaranarayanan R, Muwonge R, et al. Pooled analysis of the accuracy of five cervical cancer screening tests assessed in eleven studies in Africa and India. Int J Cancer 2008;123(01): 153-160

6 Walboomers JM, Jacobs MV, Manos MM, et al. Human papillomavirus is a necessary cause of invasive cervical cancer worldwide. J Pathol 1999;189(01):12-19

7 Muñoz N, Bosch FX, de Sanjosé S, et al; International Agency for Research on Cancer Multicenter Cervical Cancer Study Group. Epidemiologic classification of human papillomavirus types associated with cervical cancer. N Engl J Med 2003;348(06): 518-527

8 Qiagen. HC2 HIGH-RISK TEST. United States: Qiagen Gaithersburg Inc.; 2008

9 Malloy C, Sherris J, Herdman C. HPV DNA Testing: Technical and Programmatic. . Seattle, Washington, Program for Appropriate Technology in Health [http://www. popline. org]. 2000 Dec.

10 Bruni L, Diaz M, Castellsagué X, Ferrer E, Bosch FX, de Sanjosé S Cervical human papillomavirus prevalence in 5 continents: metaanalysis of 1 million women with normal cytological findings. J Infect Dis 2010;202(12):1789-1799

11 IARC Working Group on the Evaluation of Carcinogenic Risks to Humans. Human papillomaviruses. IARC Monogr Eval Carcinog Risks Hum 2007;90:1-636

12 Cuzick J, Arbyn M, Sankaranarayanan R, et al. Overview of human papillomavirus-based and other novel options for cervical cancer screening in developed and developing countries. Vaccine 2008; 26(Suppl 10):K29-K41

13 World Health Organization (WHO) Comprehensive Cervical Cancer Control. A Guide to Essential Practice Geneva: WHO; 2006

14 Rajaraman P, Anderson BO, Basu P, et al. Recommendations for screening and early detection of common cancers in India. Lancet Oncol 2015;16(07):e352-e361 
108 Cross-Sectional Study of HPV Self-Sampling among Indian Women-A Way Forward Anand et al.

15 Pimple S, Mishra G, Shastri S. Global strategies for cervical cancer prevention. Curr Opin Obstet Gynecol 2016;28(01):4-10

16 Madhivanan P, Nishimura H, Ravi K, et al. Acceptability and concordance of self-versus clinician-sampling for HPVs testing among rural south Indian women. Asian Pac J Cancer Prev 2021; 22(03):971-976

17 Sowjanya AP, Jain M, Poli UR, et al. Prevalence and distribution of high-risk human papilloma virus (HPV) types in invasive squamous cell carcinoma of the cervix and in normal women in Andhra Pradesh, India. BMC Infect Dis 2005;5:116
18 Sankaranarayanan R, Swaminathan R, Brenner H, et al. Cancer survival in Africa, Asia, and Central America: a population-based study. Lancet Oncol 2010;11(02):165-173

19 Bansil P, Wittet S, Lim JL, Winkler JL, Paul P, Jeronimo J. Acceptability of self-collection sampling for HPV-DNA testing in low-resource settings: a mixed methods approach. BMC Public Health 2014;14:596

20 Russell VL, Leeuw Sd. Intimate stories: aboriginal women's lived experiences of health services in northern British Columbia and the potential of creative arts to raise awareness about HPV, cervical cancer, and screening. J Indigenous Health 2012;8:18-27 